\title{
Primary care referral to multidisciplinary high risk foot services - too few, too late
}

D Plusch ${ }^{1}$, S Penkala ${ }^{1,4^{*}}$, HG Dickson ${ }^{2,4}$ and M Malone $e^{1,3,4}$

\begin{abstract}
Background: To determine if patients with no contact with a multi-disciplinary team High Risk Foot Service (MDT-HRFS) had an increased rate of hospital admission for diabetes foot infection compared to patients with contact. Secondary aims were to report on clinical outcomes.

Methods: A retrospective study was conducted at a major tertiary referral hospital in metropolitan Sydney over 12 months. An ICD-10 search of the electronic medical record system and paper medical charts identified patients with diabetes mellitus (type 1 or type 2) and a primary admission for diabetes foot infection (DFI). Patients were categorised as having contact or no contact with an MDT-HRFS.

Results: One hundred ninety-six hospital admissions (156 patients) were identified with DFI over a 12-month period. Patients with no contact with a MDT-HRFS represented three quarters of admissions (no contact $=116,74.7 \%$ vs. contact $=40,25.6 \%, p=0.0001$ ) and presented with more severe infection (no contact $=39 \mathrm{vs}$. contact $=12$ ). The odds of lower extremity amputation increased five-fold when both no contact and severe infection occurred in combination (no contact with severe infection and amputation $=34,82.9 \%$ vs. contact with severe infection and amputation $=7$, $17.1 \%, \mathrm{OR} 4.9,95 \% \mathrm{Cl} 1.1-21.4, p=0.037)$. Using estimates of both the contact and no contact population of people with diabetes and high-risk feet and assuming the risk of amputation was the same, than the number of expected amputations in the no contact group should have been 55 , however the observed number was 77,22 more than expected $(p=0.0001)$.
\end{abstract}

Conclusions: Patients with no contact with a MDT-HRFS represented the majority of admissions for DFIs to a tertiary referral hospital. This group on population estimates appears to be at high risk of amputation of the lower extremity and therefore early referral of this high-risk group might lower this risk.

\section{Background}

Foot infections are one of the most common causes of hospitalisation in people with diabetes $[1,2]$ with up to $85 \%$ of cases proceeding to a diabetes related lower extremity amputation [3]. Most frequently DFIs are preceded by ulceration, where a break in the protective barrier of the skin leaves a portal of entry for invading pathogenic organisms. The deficit in the immunemediated response in people with diabetes may increase the risk and severity of foot infections but the exact underlying process responsible for this deficit, remains poorly characterized [4]. Early recognition and appropriate

\footnotetext{
* Correspondence: s.penkala@westernsydney.edu.au

'Western Sydney University, Campbelltown Campus, Campbelltown, Sydney, NSW 2560, Australia

${ }^{4}$ LIVE DIAB CRU, Ingham Institute of Applied Medical Research, Liverpool,

NSW 2170, Australia

Full list of author information is available at the end of the article
}

management of diabetes related foot pathology preceding DFI's therefore is essential.

Primary care practitioners while providing the majority of medical care for people with diabetes, also play an important role in routine screening, identifying the risk of diabetes related foot pathology and referral needs [5]. High risk foot stratification includes two or more risk factors (peripheral neuropathy, peripheral vascular disease, deformity) and/or a history of ulceration, and/or amputation [6]. Clinical guidelines recommend primary referral of people with a high risk foot to a MDT-HRFS with specialist care from medical, surgical, nursing, podiatry and allied health professionals $[5,7,8]$.

Emergency referral to a MDT-HRFS within $24 \mathrm{~h}$ is recommended when there is a new ulcer, swelling or foot discolouration [8]. Grading severe infection on the Infectious Disease Society of America (ISDA) guidelines, 
predicted hospitalisation and amputation rates 89 and $70 \%$ respectively [6]. High-risk foot services have demonstrated reductions in hospital admissions [9], amputations [10-16] and length of stay (LOS) $[9,12,13,16-18]$ for patients known to the service. However, despite clinical success rates and guidelines, many people with diabetes do not receive routine screening for diabetes related foot pathology to enable appropriate early referrals [5].

While the main focus within the literature surrounds MDT-HRFS and the reduction of DRLEA rates, there has been no comparison of hospital admissions for DFIs between people with contact and no contact to a MDTHRFS. The primary aim of this study was to determine if patients with no contact to a MDT-HRFS were associated with increased numbers of hospital admission for diabetes foot infection. Secondary aims were to report on clinical outcomes in patients hospitalised for DFI, with a focus on any surgical procedures required, LOS, infection classification and cost.

\section{Methods}

A retrospective study was conducted at a major tertiary referral hospital in metropolitan Sydney from $1^{\text {st }}$ January 2012 to $1^{\text {st }}$ January 2013. Ethical approval was obtained from South Western Sydney Local Health District Research and Ethics Committee. Patient information including age, gender, medical history, clinical, laboratory, operative report data and hospital metrics were collected Patients were eligible if they had either type 1 or type 2 diabetes with a primary admission for DFI. Patients were identified using the ICD-10 coding system.

District-linked electronic medical records and paper medical charts were cross-referenced and used to identify patients with 'contact' and 'no contact' to the MDT-HRFS. Patients with contact were defined as any registered patient attending an outpatient appointment with the MDTHRFS in the preceding 12 months prior to admission. A MDT-HRFS was defined according to Agency for Clinical Innovation, Endocrine Network standards for high-risk foot services in New South Wales, Australia. There are four MDT-HRFS in the administrative district, each located in a public hospital outpatient area.

People with diabetes and peripheral neuropathy constitute a group at high risk for lower limb amputation as a consequence of ulceration and infection. While neuropathy has been identified as an etiology in the majority of DFI foot ulcerations [2, 19], peripheral vascular disease is present in over half [2] of those requiring hospitalisation and is a predictor of poor healing [2]. The size of this group in our Health District is not known, but estimates can be generated. The population of the Health District is 820,000 . Approximately $8 \%$ will have diabetes [19], and of these about $60-70 \%$ will have peripheral neuropathy and or peripheral arterial disease. The severity of the neuropathy and or peripheral arterial disease is likely to be normally distributed, and $20 \%$ could be categorized as being in the high risk group [20]. This gives a target population at high risk of diabetic foot disease of approximately 8500 people. There are approximately 2500 'known' patients on the patient register of the High Risk Foot Clinic at our hospital location. 'Unknown' patients to the High Risk Foot Clinic with high risk foot pathology are estimated to be around 6000, derived from subtracting the 'known' patients from the target at risk population of 8500 .

Outcomes of interest were: any foot surgery or vascular procedure associated with the index admission, the length of hospital stay, severity of infection (using current Infectious Disease Society of America guidelines for diabetes foot infection [2], inflammatory markers, culture results, and estimated hospital costs. Past medical history including comorbid variables in addition to clinical and laboratory data were confirmed using current diagnostic guidelines and obtained from both electronic medical records and paper charts. In particular, PAD was confirmed via documented clinical assessment and or in combination with available vascular studies. Diabetes peripheral neuropathy were confirmed through available clinical indicators including a modified neuropathic disability score $>6$ [21] absent $10 \mathrm{~g}$ monofilament in $>3$ places on the foot [22] and or available diagnostic electrophysiological studies.

Identified patients for the study were split into two cohorts, contact and no contact to a MDT-HRFS.

Costs for each inpatient separation were calculated utilizing AR-DRG (Independent Hospital Pricing Authority). All values are represented in AUS \$. For each patient, the admission, discharge date, principal diagnosis, principal procedure and co-morbidities were extracted and cross checked between electronic records and paper charts.

The cost of each individual patient episode of admission was calculated. Multiple procedures during a single admission were not calculated, and the cost was attributed to the highest surgical procedure cost. Thus if a patient required revascularization and amputation, the cost for amputation would be calculated as it is the higher costing procedure. The cost of multiple admissions within the 12-month period was also calculated for individual patients.

\section{Statistical analysis}

Statistical analysis was undertaken using IBM Statistical Packages for the Social Sciences (SPSS) Version 21.0 for Windows (SPSS Inc, Chicago, IL, USA). Unpaired t tests and chi square with risk ratio were employed in testing differences between cohorts. Mann-Whitney $U$ test was used for nonparametric data. A histogram for hospital metric data was performed to look for outliers and normality. For 
all comparisons and modelling, the level of significance was set at $p<0.05$.

\section{Results}

A total of 196 hospital admissions (156 patients) were identified from the ICD-10 search over the 12 month study period. Of the 156 patients, the majority had no contact with a MDT-HRFS (no contact $=116$, $74.7 \%$ vs. contact $=40$ patients, $25.6 \%, p=0.0001$ ). Patient characteristics including age, gender, medical history, clinical, laboratory and surgical procedures are identified in Table 1.

\section{Surgery}

A large number of patients admitted for DFI required a surgical procedure ( $n=126$ out of $156,81 \%$ ) and the majority of these were undertaken in patients with no contact ( $n=94$ out of $126,74.7 \%$ ). Amputation was the most common surgical intervention $(n=100$ out of 126 , $72 \%)$ with lower extremity amputation being undertaken more frequently in patients with no contact (no contact $=77,82 \%$ vs. contact $=23,72 \%, p=0.23$ ). Population estimates for our groups suggested that admission and amputation in the no contact group were over represented, the expected number of amputations should have been 55 , however the observed number was 77,22 more than expected $(p=0.0001)$.

Revascularisations utilising percutaneous transluminal angioplasty (PTA) were performed in $34(36 \%)$ of no contact patients and 6 contact $(19 \%)$ patients with a trend towards significance $(p=0.054)$. Regardless of contact status, digital amputations were the most commonly performed amputation $(n=67,43 \%)$

\section{Length of stay}

On average, the median LOS in the no contact group was 3 days longer than those with contact (contact = 8 days, IQR 7 to 12 vs. no contact $=11$ days, IQR 6 to $24, p=0.063)$. LOS was influenced by the requirement to undergo a surgical procedure. Regardless of contact status, the LOS for admissions not requiring surgery was 7 days (IQR 5 to 10 days) while LOS increased to 11 days in those patients undergoing a surgical procedure (IQR 8 to 21 days, $p=0.008$ ).

\section{Infection severity and classification}

IDSA moderate infection was the most common presentation regardless of contact status ( $n=105$ of $156,67 \%)$. IDSA severe infection presentations occurred in $34 \%$ ( $n=39$ of 116) of the no contact group and $30 \%$ of the contact group ( $n=12$ of $40, p=0.67$ ), however no contact patients presenting with IDSA severe infection required greater numbers of lower extremity amputation with odds of 4.9 times higher than those with contact to a
MDT-HRFS (no contact with severe infection and amputation $=34,82.9 \%$ vs. contact with severe infection and amputation $=7,17.1 \%$, OR 4.9, $95 \%$ CI 1.1 to 21.4, $p=0.037)$.

\section{Laboratory data}

Inflammatory markers were similar between groups, with no significant difference; white cell count (no contact $=12.1 \times 10^{\wedge} 9 / L$, IQR 10 to 15 vs. contact $=10.7 \times$ $10^{\wedge} 9 / \mathrm{L}$, IQR 8 to $15, p=0.241,95 \% \mathrm{CI}-0.87$ to 3.45 ), erythrocyte sedimentation rate (no contact $=57 \mathrm{~mm} /$ $\mathrm{min}, \mathrm{IQR} 26$ to 77 vs. contact $=57 \mathrm{~mm} / \mathrm{min}, \mathrm{IQR} 34$ to $86, p=0.69$ ), C-reactive protein (no contact $=76 \mathrm{mg} / \mathrm{L}$, IQR 32 to 172 vs. contact $=70 \mathrm{mg} / \mathrm{L}$, IQR 23 to 155 , $p=0.99$ ).

\section{Culture results}

Two hundred fifteen pathogens of suspected infection were isolated from soft tissue and bone cultures in 129 patients. Polymicrobial infections were more common than monomicrobial infections (monomicrobial $=44$ patients, $28.2 \%$ versus polymicrobial $=85$ patients, $54.5 \%$ ). Culture results were unavailable for 27 patients (17.3\%) who all received empiric antimicrobial therapy.

Gram-positive cocci were the predominating pathogens with the combination of Methicillin-sensitive Staphylococcus aureus (MSSA) and Methicillin-resistant Staphylococcus aureus (MRSA) accounting for half of all gram-positive cultures $(\mathrm{MSSA}=37$ of $118,31 \%$ and MRSA $=31$ of 118 , $26 \%$ ). Gram-negative bacteria represented $47 \%$ of cultures with gram-negative rods predominating as probable colonisers (71\%, 69 of 97).

\section{Cost analysis}

The estimated total cost of foot infection in this cohort was $\$ 4,264,214$. The total cost was significantly higher in the no contact $\$ 3,169,083$ primarily due to the higher rate of patient presentation and surgical intervention. In comparison, the contact group costs amounted to $97 \%$ less in total costs, $(\$ 1,095,131)$ however, the average cost per patient separation was similar between groups (no contact $=\$ 22,475$ vs. contact $=\$ 21,473, p=0.763)$.

\section{Discussion}

While evidence exists for reduction of diabetes related lower extremity amputation through the use of multidisciplinary high risk foot care services $[10,23,24]$ the link between reduction in infections and the use of multidisciplinary foot services is less clear. This retrospective study suggests access to the MDT-HRFS within the hospital at the time of admission for DFI provides similar clinical outcomes regardless of contact status prior to admission. However, in this study patients with DFI who had no previous contact with a MDT-HRFS constituted 
Table 1 Patient characteristics for contact or no contact with a MDT-HRFS

\begin{tabular}{|c|c|c|c|c|}
\hline & Contact $(n=40)$ & No Contact $(n=116)$ & Total $(n=156)$ & $p$-value \\
\hline \multicolumn{5}{|l|}{ Demographics } \\
\hline Mean Age $( \pm S D)$ & $63.4( \pm 11.8)$ & $65.5( \pm 14.1)$ & $65( \pm 13.5)$ & 0.40 \\
\hline Male, n (\%) & $27(67.5)$ & $78(67.2)$ & $105(67.3)$ & \\
\hline Female, n (\%) & $13(32.5)$ & $38(32.8)$ & $51(32.7)$ & 0.98 \\
\hline \multicolumn{5}{|l|}{ Medical history } \\
\hline Diabetes Type 1, n (\%) & $4(10.0)$ & $8(6.9)$ & $12(7.7)$ & \\
\hline Diabetes Type 2, n (\%) & $36(90.0)$ & $108(93.1)$ & $144(92.3)$ & 0.54 \\
\hline Duration of Diabetes $( \pm \mathrm{SD})$ & $15.5( \pm 5.7)$ & $15.8( \pm 8.5)$ & $15.7( \pm 7.8)$ & 0.86 \\
\hline $\mathrm{HbA} 1 \mathrm{C}(\mathrm{mmol} / \mathrm{mol}) / \mathrm{FCC}$ & $8.4( \pm 2.2) / 68$ & $8.7( \pm 2.6) / 72$ & $8.6( \pm 2.5) / 70.5$ & 0.44 \\
\hline Peripheral Neuropathy, n (\%) & $35(87.5)$ & 97 (83.6) & $132(84.6)$ & 0.56 \\
\hline Peripheral Arterial Disease, n (\%) & $33(82.5)$ & $95(81.9)$ & $128(82.1)$ & 0.93 \\
\hline Ischemic Heart Disease, n (\%) & $20(50)$ & $58(50)$ & $78(50)$ & 1.00 \\
\hline Hypertension, n (\%) & $36(90)$ & $98(84.5)$ & $134(85.9)$ & 0.39 \\
\hline CKD stage $5, \mathrm{n}(\%)$ & $9(22.5)$ & 19 (16.4) & $28(17.9)$ & 0.21 \\
\hline eGFR ( \pm SD) & $41.3( \pm 26.5)$ & $47.8( \pm 28.7)$ & $46.2( \pm 28.2)$ & $0.001^{*}$ \\
\hline Length of Stay, n (IQR) & $8(7-12)$ & $11(6-24)$ & $10(10-19.5)$ & 0.63 \\
\hline \multicolumn{5}{|l|}{ LAB } \\
\hline White Cell Count, n (IQR) & $10.75(8-15)$ & $12.1(10-15)$ & $11.8(9-15)$ & 0.24 \\
\hline Presenting ESR (mmol/L), n (IQR) & $57(34-86)$ & $57(26-77)$ & $57(27-77)$ & 0.69 \\
\hline Presenting CRP (mg/l), n (IQR) & $76.35(23-155)$ & $69.75(32-172)$ & $73.7(29-166)$ & 0.99 \\
\hline \multicolumn{5}{|l|}{ Infection severity } \\
\hline IDSA grade Moderate, n (\%) & $28(70 \%)$ & 77 (66.4 \%) & $105(67.3 \%)$ & 0.68 \\
\hline IDSA grade Severe, n (\%) & $12(30 \%)$ & 39 (33.6 \%) & $51(32.7 \%)$ & 0.68 \\
\hline \multicolumn{5}{|l|}{ Texas classification } \\
\hline $1 \mathrm{~B}, \mathrm{n}(\%)$ & $1(2.5)$ & $6(5.2)$ & $7(4.5)$ & 0.48 \\
\hline 1D, n (\%) & $6(15)$ & $21(18.1)$ & $27(17.3)$ & 0.66 \\
\hline $2 \mathrm{~B}, \mathrm{n}(\%)$ & $3(7.5)$ & $11(9.5)$ & $14(9)$ & 0.71 \\
\hline 2D, n (\%) & $12(30)$ & $52(44.8)$ & $64(41)$ & 0.10 \\
\hline $3 B, n(\%)$ & $3(7.5)$ & $5(4.3)$ & $8(5.1)$ & 0.43 \\
\hline 3D, n (\%) & $15(37.5)$ & $21(18.1)$ & $36(23.1)$ & $0.01^{*}$ \\
\hline \multicolumn{5}{|l|}{ Surgery type } \\
\hline Surgical debridement, n (\%) & $7(17.5)$ & $28(24.1)$ & $35(22.4)$ & 0.39 \\
\hline PTA, n (\%) & $6(15)$ & $34(29.3)$ & $40(25.6)$ & $0.08^{*}$ \\
\hline Bypass, n (\%) & $0(0)$ & $3(2.6)$ & $3(1.9)$ & 0.31 \\
\hline Amputation, n (\%) & $23(57.5)$ & $77(66.4)$ & $100(64.1)$ & 0.32 \\
\hline Forefoot/digital, n (\%) & $16(40)$ & $51(44)$ & $67(42.9)$ & 0.66 \\
\hline TMA, n (\%) & $0(0)$ & $0(0)$ & $0(0)$ & \\
\hline BKA, n (\%) & $8(20)$ & $18(15.5)$ & $26(16.7)$ & 0.51 \\
\hline AKA, n (\%) & $0(0)$ & $8(6.9)$ & $8(5.1)$ & $0.09^{*}$ \\
\hline
\end{tabular}

ESR erythrocyte sedimentation rate, CRP c-reactive protein, CKD chronic kidney disease, eGFR estimated glomelular filtration rate, PTA percutaneous transluminal angioplasty 
around three times the number of hospital admissions for lower extremity amputation compared to those with prior contact. Whilst this rate of lower extremity amputation was not significantly different between the two groups, population estimates suggest that the number of expected amputations in the no contact group should have been 55 , however the observed number was 77,22 more than expected.

The large numbers of patients with no contact to MDTHRFS within a large district in Sydney, Australia is of concern, particularly with four services in our District, with a no-wait policy for urgent referrals. Population estimates suggest this group are over represented in relation to admission and amputation rates. Screening is the key to identify people with diabetes who are at risk of ulceration and complication [25]. This has been emphasised in many health policy documents $[7,8]$. A large component of this work is often undertaken within the primary care setting. Whilst the baseline characteristics of the contact and no contact group were similar, it is surprising given the long standing duration of diabetes and co-morbidities that referral to a MDT-HRFS did not routinely occur.

Our results are consistent with the contention that MDT-HRFS reduce the risk of amputation particularly in association with severe infection. Severe DFI was associated with a five-fold increase in the odds of requiring a lower extremity amputation. Using the same criteria for grading severity of infection as in this study, Wukich and colleagues evaluated the outcomes of patients with moderate and severe DFI. Their retrospective study of 119 patients reported a similar seven-fold increase in the risk of patients undergoing amputation if they presented with severe DFI (7.12 RR $95 \%$ CI 1.83-41.05) [26].

The median LOS in patients with no contact was three days longer than those with contact. While this was not significant between the groups the additional hospital days are associated with an increased burden on the healthcare system and this number is higher than national averages for admission with cellulitis without complications [27]. The most likely explanation for this finding is the higher number of surgical procedures undertaken during admission in this study population.

This study has limitations that should be noted. The retrospective design relies heavily on both the ability of the treating team and the clinical coding team to accurately capture all relevant patient data and assign a correct primary diagnosis [26]. Errors may also occur in the ICD-10 conversion to the correct DRG [28]. A prospective study would allow greater accuracy in coding and classification.

The AR-DRG clinical coding which is used to estimate healthcare costs can be influenced by under and overcoding and by coding errors. In this study, these problems were avoided by allocating AR-DRG codes for each submission after manually cross-referencing all of the data between the ICD-10 search, eMR and patient paper charts, rather than relying solely on the patient paper charts.

\section{Conclusion}

This single-centre study indicates that patients with no contact with a multi-disciplinary high risk foot service account for around $75 \%$ of hospital admissions and amputations for DFI. Given the evidence about the effectiveness of MDT-HRFS and the need for early identification and prevention the over representation of the no contact group is of concern. Appropriate referral and early access to these specialist clinics is needed. Primary health carers and general practitioners should be aware of patients that should be referred to MDT-HRFS. Future studies should be prospective and a multi-site study would provide a national perspective. Studies should also explore the potential barriers to early referral for those at risk of admission for complications of diabetes foot disease.

\section{Competing interests}

No relevant disclosures.

\section{Authors' contributions}

DP collected data, designed data collection forms and undertook a literature review for the introduction. MM and SP undertook statistical analysis and provided input into the first draft of the article. HGD, MM and SP were responsible for the final draft paper. All authors read and approved the final manuscript.

\section{Author details}

${ }^{1}$ Western Sydney University, Campbelltown Campus, Campbelltown, Sydney, NSW 2560, Australia. ${ }^{2}$ Ambulatory Care, Liverpool Hospital, Locked Bag 7103, Liverpool, NSW 2170, Australia. ${ }^{3}$ Department of Podiatric Medicine, High Risk Foot Service, Liverpool Hospital, Locked Bag 7103, Liverpool, NSW 2170, Australia. ${ }^{4}$ LIVE DIAB CRU, Ingham Institute of Applied Medical Research, Liverpool, NSW 2170, Australia.

Received: 30 August 2015 Accepted: 1 November 2015

Published online: 14 November 2015

\section{References}

1. Kruse I, Edelman S. Evaluation and treatment of diabetic foot ulcers. Clin Diabetes. 2006;24(2):91-3.

2. Lipsky BA, Berendt AR, Cornia PB, Pile JC, Peters EJG, Armstrong DG, et al. Infectious disease society of America clinical practice guideline for the diagnosis and treatment of diabetic foot infections. Clin Infect Dis. 2012;54(12):132-73.

3. Boulton AJM. The diabetic foot. Medicine. 2010;38(12):644-8.

4. Gupta S, Koirala J, Khardori R, Khardori N. Infections in Diabetes Mellitus and Hyperglycemia. Infect Dis Clin North Am. 2007;21:617-38.

5. The Royal College of General Practitioners. General practice management of type 2 diabetes 2014-2015. Melbourne: The Royal College of General

Practitioners; 2014.

6. Lavery LA, Armstrong DG, Murdock DP, Peters EJ, Lipsky BA. Validation of the infectious disease society of America's diabetic foot infection classification system.

Clin Infect Dis. 2007:44:562-5.

7. National Health and Medical Research Council. National Evidence-Based Guideline on Prevention, Identification and Management of Foot Complications in Diabetes: Part of the Guidelines on Management of Type 2 Diabetes. Melbourne: National Health and Medical Research Council; 2011. p. 56. 
8. National Institute for Clinical Excellence. Type 2 diabetes foot problems: Prevention and management of foot problems. 2011.

9. Lavery LA, Wunderlich RP, Tredwell JL. Disease management for the diabetic foot: Effectiveness of a diabetic foot prevention program to reduce amputations and hospitalizations. Diabetes Res Clin Pract. 2005;70:31-7.

10. Krishnan S, Nash F, Baker N, Fowler D, Rayman, G. Reduction in diabetic amputation over 11 years in a defined U.K.population. Diabetes Care. 2008:31(1):99-101.

11. Apelqvist J, Larsson J. What is the most effective way to reduce incidence of amputation in the diabetic foot? Diabetes Metab Res Rev. 2000;16 Suppl 1:S75-83.

12. Nather A, Bee CS, Lin WK, Valerie CXB, Liang S, Tambyah PA, et al. Value of team approach combined with clinical pathway for diabetic foot problems: a clinical evaluation. Diabet Foot Ankle. 2010. doi:10.3402/dfa.v110.5731.

13. Anichini R, Zechinni F, Cerretini I, Meucci G, Fusilli D, Alviggi L, et al. Improvement of diabetic foot care after the implementation of the International Consensus on the Diabetic Foot (ICDF): results of a 5-year prospective study. Diabetes Res Clin Pract. 2007;75:153-8.

14. Canavan RJ, Unwin NC, Kelly WF, Connolly VM. Diabetes and non diabetesrelated lower extremity amputation incidence before and after the introduction of better organised diabetes foot care. Diabetes Care. 2008:31:459-63.

15. Edmonds ME, Blundell MP, Morris ME, Thomas EM, Cotton LT, Watkins PJ. Improved survival of the diabetic foot: the role of a specialised foot clinic. $Q$ J Med. 1986;60:763-71.

16. Mclnnes AD. Diabetic foot disease in the United Kingdom: about tome to put feet first. J Foot Ankle Res. 2012;5(26):1-7.

17. Leichter SB, August GL, Moore W. The business of hospital care of diabetic patients: 2. A new model for inpatient support services. Clin Diabetes. 2003;21:136-9.

18. Rubin RJ, Dietrich KA, Hawk AD. Clinical and economic impact of implementing a comprehensive diabetes management program in managed care. J Clin Endocrinol Metab. 1998;83:2635-42.

19. Boulton AJ. The diabetic foot: neuropathic in etiology. Diabet Med. 1990;7:852-8.

20. Tapp RJ, Shaw JE, de Courten MP, Dunstan DW, Welborn TA, Zimmet PZ Foot complications in Type 2 diabetes: an Australian population-based study. Diabetes Med. 2003;20(2):105-13.

21. Abbott CA, Carrington AL, Ashe H, Baths S, Every LC, Griffiths J, et al. The North-West diabetes foot care study: incidence of, and risk factors for, new diabetic foot ulceration in a community-based patient cohort. North-West Diabetes Foot Care Stud Diabetes Med. 2002;19(5):377-84.

22. American Diabetes Association. Preventative foot care in people with diabetes: A consensus statement. Diabetes Care. 2004;27 suppl 1:s63-4.

23. Holstein P, Ellitsgaar N, Bornefeldt O, Ellitsgaard, V. Decreasing incidence of major amputations in people with diabetes. Diabetologia. 2000;43:844-7.

24. Lavery LA, Armstrong DG, Wunderlich RP, Mohler MJ, Wendel CS, Lipsky BA. Risk factors for foot infections in individuals with diabetes. Diabetes Care. 2006;29(6):1288-93.

25. Pickwell K, Siersma V, Kars M, Apelqvist J, Bakker K, Edmonds M. Predictors of Lower-extremity amputation in patients with an infected diabetic foot ulcer. Diabetes Care. 2015. doi:10.2337/dc14-1598.

26. Wukich DK, Hobizal KB, Raspovic KM, Rosario BL. SIRS is valid in discriminating between severe and moderate diabetic foot infections. Diabetes Care. 2013;36:3706-11.

27. National Health Performance Authority 2013, Hospital Performance: Length of stay in public hospitals in 2011-12. www.nhpa.gov.au.

28. Daultrey H, Gooday C, Dhatariya K. Increased length of inpatient stay and poor clinical coding: audit of patients with diabetes. J Royal Soc Med Short Reports. 2011;2(83):1-6.

\section{Submit your next manuscript to BioMed Central and take full advantage of:}

- Convenient online submission

- Thorough peer review

- No space constraints or color figure charges

- Immediate publication on acceptance

- Inclusion in PubMed, CAS, Scopus and Google Scholar

- Research which is freely available for redistribution

Submit your manuscript at www.biomedcentral.com/submit 